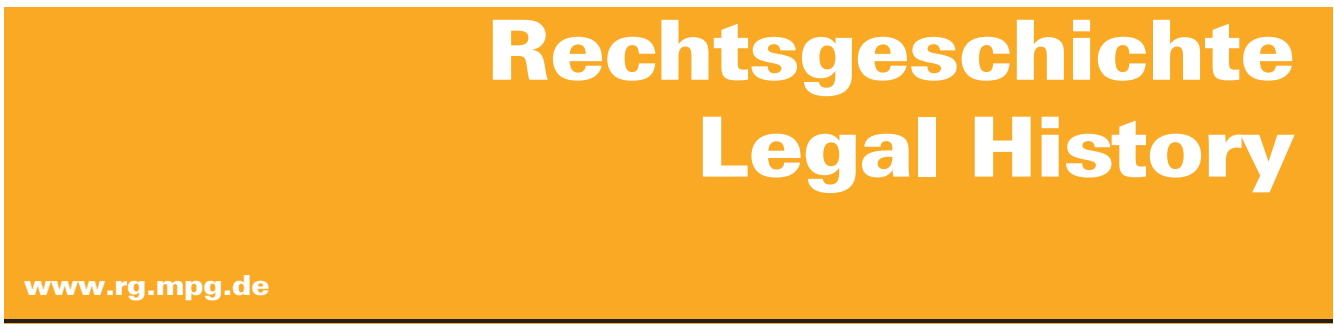

http://www.rg-rechtsgeschichte.de/rg23

$\operatorname{Rg} 232015$

$322-325$

Zitiervorschlag: Rechtsgeschichte - Legal History Rg 23 (2015)

http://dx.doi.org/10.12946/rg23/322-325

\title{
Claudia Curcuruto
}

»Pecunia nervus rerum«: Justitia als Merkurs hörige Schwester 
dargeboten: »Die spezielle Frage nach Gastfreundschaft und die folgende Recherche von unterschiedlichen Quellen nach Aussagen zu diesem Thema hat ein in seiner Bedeutung weit über dieses Thema hinausreichendes Ergebnis erbracht: Für die AkteurInnen war die frühneuzeitliche Gesellschaft eine Haushaltsgesellschaft« (482). Man weiß zwar ungefähr, was gemeint ist, aber diesem
Standard genügen bereits nachlässig übersetzte Gebrauchsanweisungen für chinesische Toaster.

Schade, dass die Verfasserin die reiche Gabe ihrer Quellenauswahl und -wiedergabe nicht noch um das Geschenk einer mutigen, eigenständigen Analyse vermehrt hat.

\section{Claudia Curcuruto}

\section{»Pecunia nervus rerum«: Justitia als Merkurs hörige Schwester*}

Die Beschäftigung mit geistlichen Territorien des frühneuzeitlichen Reiches erlebt seit 2003 eine besondere Aufmerksamkeit und Beliebtheit. ${ }^{1}$ Besonders in der aktuellen Forschung kann durch die Aufnahme neuer methodischer Ansätze und vielfältiger Perspektiven von einem »return« der Diskussion um die Propria geistlicher Staaten gesprochen werden. Ein solcher perspektivischer Neuansatz liegt mit der Monographie von Michael Ströhmer aus dem Jahre 2013 vor. So fordert der Frühneuzeithistoriker eine tiefgreifende »Neubewertung geistlicher Landesstaaten« (15), um historiographische Negativbeurteilungen geistlicher (katholischer) Staatlichkeit durch aufgebürdete Zuschreibungskategorien wie "rückständig" oder »retardierend « im Gegensatz zum weltlichen (protestantischen) Fürstenstaat erneut zu hinterfragen. Ausgehend von dieser Problematik postuliert Ströhmer dagegen eine Dekonstruktion des Fortschrittsparadigmas. Die Studie verfolgt aus diesem Grunde das Ziel einer differenzierteren »Identifizierung geistlicher Staatlichkeit« (18) als bisher geschehen und beabsichtigt hierbei den frühneuzeitlichen Staatsbildungsprozess im Justizsektor adäquat zu charakterisieren $(12,18,20)$.

Das Rekonstruktionsmodell von geistlicher Staatlichkeit und Muster eines frühneuzeitlichen Staatsbildungsprozesses, die der Frühneuzeithistoriker mit einer großen empirischen Untersuchung dem Leser anbietet, ist das Konzept der Jurisdiktionsökonomie. Es ist ein theoretischer interdisziplinärer Neuansatz, der von der »Neuen Institutionsökonomik « (NIÖ) inspiriert ist und im Grunde als eine wirtschaftstheoretische Erweiterung von Justiznutzung beschrieben werden kann. Die methodologische Form der Untersuchung erfolgt auf induktivem Wege durch einen »Ansatz von unten« (»Bottom-up-Modell«), um auf diese Weise einen zentralen Herrschafts- und Rechtsraum der Frühen Neuzeit primär aus der Nutzerperspektive der Stiftsuntertanen zu rekonstruieren sowie die Interventionsmöglichkeiten der einzelnen Justiznutzer bei der Gestaltung des frühneuzeitlichen Justizsystems darzulegen. Dadurch erst ist es nach Ströh-

\footnotetext{
* Michael Ströhmer, Jurisdiktionsökonomie im Fürstbistum Paderborn. Institutionen - Ressourcen Transaktionen (1650-1800) (Westfalen in der Vormoderne. Studien zur mittelalterlichen und frühneuzeitlichen Landesgeschichte 17), Münster: Aschendorff 2013, 376 S., ISBN 978-3-402-15057-3
}

\footnotetext{
1 Grundlegend ist hierfür insbesondere ein Sammelband, der die geistlichen Staaten anlässlich der Gedenktage zum 200. Jahrestag der Säkularisation wieder nachhaltig in Erinnerung brachte, vgl. Braun, Bettina u. a. (Hg.), Geistliche Staaten im Nordwesten des Alten Reiches. Forschungen zum Problem frühmoderner Staatlichkeit, Köln 2003.
} 
mer möglich, den »historischen Blick auf alternative Deutungen von frühmoderner Staatswerdung zu schärfen« (20).

Die sprichwörtliche Wendung »das Geld ist der Nerv der Dinge ${ }^{2}$ drückt dabei die synergetische Grundkonzeption der Jurisdiktionsökonomie aus: Dabei gestaltet sich die frühneuzeitliche Gerichtslandschaft als ein Jurisdiktionsmarkt, auf dem Anbieter (Gerichtsherrn, Richter) und Nachfrager (Kläger, Anzeiger) aufeinandertreffen. Die Nachfrager haben aufgrund der Anbieterkonkurrenz (Landesherr, Stände, Kirche) die Möglichkeit zwischen den Angeboten der Tribunale die für sie günstigste Option auszuwählen. Die Anbieterseite wiederum ist infolge des institutionellen Wettbewerbs daran interessiert, etwa zwecks Herrschaftssicherung und Autoritätsanspruch auf dem Justizmarkt, mit den Justiznutzer in einen Aushandlungsprozess (»negotiating«, 17) zu treten. Auf diese Weise kommt es auf dem Jurisdiktionsmarkt zu permanenten Transaktionen von Rechtsgütern sowie einem Interessenausgleich zwischen Anbieter und Nachfrager. In diesem Austauschprozess von Rechtsgütern entstehen jedoch auf beiden Seiten Transaktionskosten. Insbesondere auf der Nachfragerseite dürften die »Marktnutzungskosten« (Verfahrensdauer, Mobilitätskosten zum Gerichtsstandort, die Effektivität der Entscheidung) das Nachfrageverhalten der Justiznutzer bei der Wahl des Anbieters auf dem Markt signifikant beeinflusst haben. So zunächst der theoretische Ansatz der Jurisdiktionsökonomie von Michael Ströhmer, um die Spezifika der Rechtskultur geistlicher Staaten zu umreißen (18). Die empirische Studie konzentriert sich dabei räumlich infolge ihrer gleichartig strukturierten Gerichtslandschaft auf den nordwestlichen Justizraum von »Fünfkirchen« (Kurköln, Münster, Osnabrück, Paderborn, Hildesheim), im Wesentlichen jedoch auf das geistliche Fürstentum Paderborn beziehungsweise auf die Jurisdiktionspolitik des Oberamtes Dringenberg als dessen lokalen Referenzraums. Zeitlich befasst sich die Untersuchung entsprechend dem räumlichen Zuschnitt mit den Regie- rungen der Paderborner Fürstbischöfe zwischen 1650 und 1800 .

Mithilfe des Analysemodells der Jurisdiktionsökonomie beabsichtigt Ströhmer nun das Forschungsparadigma monopolitischer Staatsbildung für den geistlichen Staat zu relativieren. Dafür schlägt der Frühneuzeithistoriker drei Aspekte vor (multiperspektivische Raumanalyse, Transaktionskostenanalyse und Jurisdiktionspolitik), die auch seiner thematischen Gliederung und seinen Ergebnissen folgt: In der ersten Untersuchungseinheit »Topographische Skizzen einer stiftischen Gerichtslandschaft des 18. Jahrhunderts« (33-127) unterstreicht Ströhmer in einer multiperspektivischen Raumanalyse anhand des gewählten DreiEbenen-Modells um die Mikro- (Oberamt Dringenberg), Meso- (Fünfkirchen) und Makroebene (Germania Sacra) die Relevanz des topographischen Arguments für die frühneuzeitliche Justiznutzung durch Einzelakteure, zunächst jedoch basierend auf einer regionalen und lokalen Ebene. Der zweite Abschnitt (128-170) befasst sich mit der Verschiebung der Justizlandschaft hin zum Jurisdiktionsmarkt und thematisiert die Wahl des Gerichtes durch den einzelnen Justiznutzer und die verfügbaren Optionen, die den Prozessparteien zu Verfügung stehen. Der Frühneuzeithistoriker wendet im dritten Schritt (171-319) schließlich das Analysemodell der Jurisdiktionsökonomie auf den spezifischen Justizraum des Fürstbistums Paderborn mit Fokus auf dem Verhalten und den Bedürfnissen der verschiedenen Akteure, den Rechtsgütern im Aushandlungsprozess zwischen Anbieter und Nachfrager sowie den Ressourcen und Kosten auf dem Jurisdiktionsmarkt anhand einer quantifizierenden Transaktionskostenanalyse. Im vierten Abschnitt »Jurisdiktionspolitik zwischen Marktmacht und Machtmonopole« (320348) legt Ströhmer die verschiedenen politischen Handlungsspielräume der fürstbischöflichen Administration und die Möglichkeiten der Gestaltung einer stiftischen Jurisdiktionspolitik in verschiedenen territorialen und soziokulturellen Einrichtungen dar.

2 Zur Herkunft und zu den Bedeutungszusammenhängen der antiken Sentenz vgl. Stolleis, Michael, Pecunia nervus rerum. Zur Staatsfinanzierung in der frühen Neuzeit, Frankfurt am Main 1983, 63-68. 
Aus den empirischen Befunden der drei Darstellungseinheiten kommt Ströhmer schließlich zu dem Ergebnis, dass der permanente Aushandlungsprozesses von Rechtsgütern und die pluralistische Gerichtsorganisation sowohl für die Stiftsbevölkerung wie auch für den Gerichtsherrn profitabel waren. Der Autor relativiert in seiner Analyse auf zwei Ebenen den immer wieder vorgebrachten Vorwurf der "Strukturschwächen « geistlicher Justizadministration (319) durch die mikrohistorischen Strukturbefunde am Beispiel des Oberamtes Dringenberg, in Analogie zur makrohistorischen Jurisdiktionsökonomik des nordwestdeutschen Großraumes: Die erste Ebene konzentriert sich auf die topographischen wie finanziellen Determinanten bei der Wahl eines Gerichtes durch den Justiznutzer. So war der Standort auf regionaler, lokaler Ebene (Binnengerichte) infolge der Dichte der Gerichtsorganisation auf dem Jurisdiktionsmarkt »nutzerfreundlicher« (350) und damit mit einem erheblich geringeren persönlichen Kostenrisiko verbunden als die Nutzung der weiträumigen Amtsjurisdiktion des Landesherrn. Die zweite Diskursebene zentralisiert sich auf den lokalgerichtlichen Kompetenzwettbewerb auf dem Jurisdiktionsmarkt des Nordwestens des Reiches. So weist der Autor darauf hin, dass bis zum Ende des Reiches die Jurisdiktionspolitik durch den geistlichen (Paderborner) Fürstbischof in seiner Rolle eines "Wettbewerbshüters" und nicht durch den eines souveränen Monopolisten gefördert wird (351). Im Gegensatz zu den zentralistisch geführten geistlichen Staaten wie den Hochstiften Mainz oder Trier liegen die Spezifika norddeutscher stiftischer Jurisdiktionspolitik nach Ströhmer demnach in der weitgehenden Aufrechterhaltung der kommunalen Selbstverwaltung und ständischen Jurisdiktionsgewalt sowie im "sozioökonomischen Konsens « zwischen Fürstbischof/Gerichtsherr und der Mitbestimmung der Justiznutzer. Eine These freilich, wie Ströhmer zugeben muss, die noch weiterer Feldstudien ähnlichen Zuschnitts bedarf, um sie auf eine breitere Grundlage zu stellen (319).
Ob nun "Justitia nur Merkurs hörige Schwester" ist (11, 353), kann der Autor weder eindeutig bejahen noch verneinen (das ist auch nicht seine Absicht). Ohne Weiteres trägt Ströhmer jedoch dazu bei, einer weiteren Disqualifizierung der (norddeutschen) geistlichen Staaten im Rahmen frühneuzeitlicher Staatsbildung durch neue Perspektiven (vor allem 349-353) zu begegnen und die »Fesseln des Schlendrians ${ }^{3}{ }^{3}$ innerhalb der gefassten Forschungsmeinungen zur Rückständigkeit geistlicher frühneuzeitlicher »frühmoderner« Staaten zu zerbrechen. Damit leistet der Band einen wichtigen Beitrag zur längst noch nicht abgeschlossenen Erforschung der Propria der geistlichen Staaten im Heiligen Römischen Reich. Weiterhin bewährt hat sich auf diesem Feld zudem die interdisziplinäre Zusammenarbeit der Geschichts- und Rechtswissenschaft mit der Sozial-, Finanz- und Wirtschaftsgeschichte. Zudem fällt das Werk durch seine Ausstattung mit zahlreichen Abbildungen und Tabellen auf (Übersicht auf den Seiten 375-376), ein Personen- und Ortsregister fehlt bedauerlicherweise.

Michael Ströhmer konnte mit dieser Studie die Rekonstruktion eines juridischen Systems geistlicher Staaten "von unten «, wie vorgeschlagen wird, darbieten, doch ist es ihm infolge der Partikularität des Rechts und der Gerichtsbarkeit nicht gelungen ein Gesamtbild vorzulegen. Damit ist bereits ein Grundproblem der Studie angesprochen, das der »disparaten Quellenlage« (292). Wie der Autor selbst zugibt, werden in der empirischen Auswertung des überlieferten Aktenmaterials nur indirekte Zugänge zu den Handlungsmotiven und Entscheidungen von Justiznutzern für die Darstellung eines Zusammenhanges von Ökonomie und Prozessstrategie eruiert. Dabei versucht er über quantifizierende Strukturanalysen, über statistische Erfassungen und binnenräumliche Vergleiche diese freizulegen, wobei »nur partielle Eindrücke« (292) gewonnen werden.

Ein weiteres Problem liegt im gewählten $\mathrm{Zu}$ schnitt des »Drei-Ebenen-Modells«. Im Grunde

3 Diesen Ausspruch hatte das Westfäli-

sche Landesmuseum zum Aufhänger

seiner Ausstellung zum Säkularisie-

rungsjubiläum gewählt: WeIss,

Gisela/Gerd Dethless (Hg.),

Zerbrochen sind die Fesseln des

Schlendrians. Westfalens Aufbruch

in die Moderne, Münster 2002. 
präsentiert sich die Studie als eine Analyse eines spezifischen regionalen Rechtsraumes. Einerseits gelingt es Ströhmer die makrohistorischen Befunde im »kleinen Raum« am Beispiel des Oberamtes Dringenberg mit Erfolg zu verifizieren, andererseits gerät das Gesamtsystem kaum in den Blickwinkel. Der Autor unterlässt es die Ergebnisse in einen größeren Zusammenhang einzuordnen, die Makroebene (Germania Sacra) wird allenfalls gestreift. Gerade die lokale Engführung und die ausschließliche Konzentration auf lokale Quellen auf allen Raumebenen machen es schwierig, die »Evidenz des räumlichen Arguments« (121) und damit das Modell der Jurisdiktionsökonomie in einem größeren Ordnungs- und Raumzusammenhang anzuwenden. Schade ist auch, dass eine ernsthafte Beschäftigung mit der »Komplexität des Systems« bzw. dem "Gewirr der Strukturen« (43) unter Beachtung des »exterritorialen Überbaus«, wie den Verfahren beim Reichskammergericht und dem Reichshofrat sowie der Einbindung der Fürstbischöfe in die bischöflich-päpstliche Hierarchie, nicht unternommen wurde. Weiterhin ist das Wir-

\section{Johannes Liebrecht Teile ohne Ganzes*}

Wie in der US-amerikanischen law and literature-Bewegung gehen seit Jahren auch hierzulande viele Juristen dem spannenden Wechselbezug von Recht und Literatur nach. In den philologischen Wissenschaften wird dem Themenbereich ebenfalls Aufmerksamkeit geschenkt, und seine Spielarten sind zahlreich. Zu glauben, dass es dabei interdisziplinär zugehen müsse, wäre jedoch Selbsttäuschung. Die beteiligten Literaturwissenschaftler interessieren sich so gut wie nie für das eigentlich rechtswissenschaftliche Bild vom betroffenen Recht, andererseits sind auch für Juristen die ken des Tribunals der Apostolischen Nuntiatur nicht in Betracht gezogen worden, die ebenso in der stark angefochtenen Zivil- und Kriminalitätsgerichtsbarkeit in zweiter bzw. dritter Instanz, also nicht ausschließlich in geistlichen Sachen (52), auf der regionalen Ebene wirken kann und für den Justiznutzer ebenso Appellationsinstanz in weltlichen Zivilsachen war. Zudem hätte die Beachtung der »römischen « Quellen dem topografischen Argument innerhalb des Jurisdiktionsmarktes zusätzliche Schärfe verleihen und damit weitere Kontaktpunkte innerhalb der norddeutschen Territorien mit ihren Verästelungen erfassen können. Überhaupt wird der Stellenwert der römischen Kurie und ihre Wirkungszusammenhänge auf der lokalen Ebene nicht deutlich. Weitere Studien eines Ineinanders von diachronen und raumübergreifenden Studien müssen nun zeigen, ob der eruierte Neuansatz nicht tatsächlich einen Sonderfall darstellt. literarischen Blickerweiterungen kaum von irgend nennenswerter, echter Relevanz. ${ }^{1}$ Das Lamento, beide Perspektiven schrieben aneinander vorbei und nähmen die je andere nie hinreichend auf, kehrt immer wieder. Dass die Erwartung von Interdisziplinarität gleichwohl weiterhin gehegt wird, irritiert dabei etwas, schließlich handelt es sich bei law as oder auch in literature nicht eigentlich um die Begegnung zweier Wissenschaften.

Einer solchen Erwartung scheint auch der von Claudia Lieb und Christoph Strosetzki herausgegebene Sammelband anzuhängen. Einerseits möchte

\footnotetext{
Claudia lieb, Christoph Strosetzki (Hg.), Philologie als Literatur- und Rechtswissenschaft. Germanistik und Romanistik 1730-1870, Heidelberg 2013, 286 S., ISBN 978-3-8253-5913-3
} 